Tab. 1

Characteristic Cases of the Albumin Turbidity Reaction

The table presents cases of the lowest, mean and highest observed values of the reaction in correlation to the serum protein levels and some turbidity reactions findings of blood serum

\begin{tabular}{|c|c|c|c|c|c|c|c|c|}
\hline Case No. & Sex, Age, Diagnosis & ATR & T. P. & S. A. & A/G & Gr & $\mathrm{Ku}$ & $\mathrm{Ta}$ \\
\hline $\begin{array}{l}1 \\
2 \\
3 \\
4 \\
5 \\
6 \\
7 \\
8\end{array}$ & 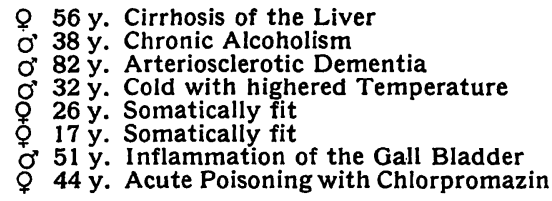 & $\begin{array}{r}0,9 \\
1,6 \\
1,8 \\
10,0 \\
21,6 \\
50,0 \\
\text { over } 50,0 \\
\text { over } 50,0\end{array}$ & $\begin{array}{l}6,80 \\
7,15 \\
4,64 \\
6,75 \\
7,60 \\
7,75 \\
7,60 \\
7,72\end{array}$ & $\begin{array}{l}2,40 \\
2,97 \\
1,85 \\
4,25 \\
4,40 \\
5,20 \\
3,70 \\
4,01\end{array}$ & $\begin{array}{l}0,55 \\
0,71 \\
0,66 \\
1,70 \\
1,37 \\
2,04 \\
0,95 \\
1,08\end{array}$ & $\begin{array}{r}1,9 \\
6,1 \\
13,8 \\
7,9 \\
5,5 \\
2,9 \\
9,6 \\
4,1\end{array}$ & $\begin{array}{r}16,2 \\
5,0 \\
2,2 \\
3,4 \\
4,6 \\
4,0 \\
6,7 \\
5,2\end{array}$ & $\begin{array}{l}++ \\
= \\
E\end{array}$ \\
\hline
\end{tabular}

Abbreviations

ATR: Albumin Turbidity Reaction (in MACLAGAN turbidity units)

T.P.: Total Serum Proteins (in $\mathrm{g} / 100 \mathrm{ml}$ )

S. A.: Serum Albumin (in $\mathrm{g} / 100 \mathrm{ml}$ )

A/G: Albumin/Globuline Quotient

Gr: GREENSPAN Test ("Acid Precipitable Globuline") in MACLAGAN turbidity. unit

Ku: Zincsulphate Test of KUNKEL (in MACLAGAN turbidity units)

Ta: Takata Reaction

albumin binding - by phenothiazin compounds in cases of ataracties intoxication and by fatty acids in cases of the gall ducts obstruction. Very high values of the albumin turbidity reaction must be therefore always evaluated taking into consideration the serum albumin level findings, further the laboratory findings of the gall ducts obstruction and the results of the toxicologic examination. On the other hand, low values of the albumin turbidity reaction uniformly show the presence of a pathologic condition. This is why the method described in this paper, is owing to its simplicity and quick employment possibilities, a suitable screeningtest of pathologic anomalies of the serum albumin, hypoalbuminemia, and of the lowered albumin/globuline quotient as well.

Typical cases of findings of the albumin turbidity reaction in relation to other indices are seen in table 1 .

\title{
References
}

1. Aokr, K. and J. F. Foster, J. Am. Chem. Soc. 78, 3538 (1956). - 2. Foster, J. F., M. Sogami, H. A. Petersen and W. J. Leonard jR., J. Biol. Chem. 240, 2495 (1965). - 3. PeterSEN, H. A. and J. F. Foster, J. Biol. Chem. 240, 2503; 3858 (1965). - 4. Shank, R. E. and C. L. Hoagland, J. Biol. Chem.
162, 133 (1946). - 5. Arbeitsvorschriften für das Pulfrich-Photometer, Sammlung I, Carl Zeiss, Jena, Gustav Fischer Verlag, Jena 1959, p. 183. - 6. Greenspan, E. M., J. Mt. Sinai Hosp., 21, 270 (1955). - 7. Kunked, H. G., E. H. Ahrens and N. J. EISENMENGER, Gastroenterology 11, 499 (1948).

Dr. St. Turek

Prag 4/CSSR, Olbrachtova 1057

\section{Untersuchung von Harnsteinen mit Hilfe eines neuen analytischen Verfahrens}

\author{
Von G. LIPTAY und M. BERENYI
}

\begin{abstract}
Aus dem Institut für Allgemeine Chemie der Tecbnischen Universität Budapest (Direktor: Prof. Dr. L. Erdey) und der Urologischen Klinik der Universität für Medizinische Wissenschaften Budapest (Direktor: Prof. Dr. A. Babics)
\end{abstract}

(Eingegangen am 2. November 1966)

Es wird ein neues komplexes, thermoanalytisches Instrument, der Derivatograph, zur Prüfung von Nierensteinen vorgeschlagen. Die meisten Komponenten der Steine erleiden bei steigender Temperatur verschiedene physikalische und chemische Änderungen. Aus der Temperatur bei der diese Änderungen auftreten bzw. aus dem Maß der Gewichtsänderung kann die Art und Menge der betreffenden Komponenten gefolgert werden. Die Methode ist sowohl für Forschungszwecke, wie für Routineuntersuchungen geeignet.

A new complex thermoanalytical instrument, the derivatograph, is proposed for testing kidney stones. Most components of the stones undergo various physical and chemical changes with increasing temperature. The type and quantity of the constituents can be determined from the temperature at which these changes occur and from the change in weight. The method is suitable for both research and routine investigations.

Die sog. Thermoanalyse zeigt gewisse vorteilhafte Eigenschaften bei ihrer Anwendung in der klinischen Analyse. Zur Bestimmung der Zusammensetzung von Harnsteinen kann man sich vielerlei analytischer Methoden bedienen. Wir benutzten zur Bestimmung der kristallinen Komponenten ein neues, an der Technischen Universität Budapest konstruiertes komplexes thermoanalytisches Gerät, den sog. Derivatographen. Die Thermoanalyse untersucht die auf Einwirkung von Temperaturänderungen in den verschiedenen Stoffen verlaufenden Vorgänge. Die meisten Substanzen, darunter die Nierensteine erfahren bei gleichmäßig steigender Temperatur verschiedene physikalische und chemische Änderungen, aus denen man die qualitative und quantitative Zusammensetzung der Komponenten der betreffenden Substanz folgern kann. Der Derivatograph nach F. PAúlik, J. PAulik und L. ERDEy registriert als Funktion der Temperatur der Probe die Crewichtsänderungen (TG-Kurve), die Geschwindigkeit der Gewichtsänderung (DTG-Kurve), weiterhin die Enthalpieänderung (DTA-differential thermoanalytische Kurve) der zu prüfenden Substanz. 
Die schematische Skizze des Gerätes ist in Abbildung 1 dargestellt. Wie zu sehen ist, besteht es aus einer analytischen Waage, deren senkrecht verlängerter Balken in den Luftraum eines Ofens reicht. Daran befindet sich ein entsprechender Behälter mit der Probe, in unserem Fall mit dem Nierenstein. Die Temperatur wird in der Einbuchtung des Probe-Behälters mit Hilfe eines Thermoelements gemessen. Das jeweilige Gewicht der Probe wird durch Projektierung der Bewegung des Waagebalkens registriert; die Derivierte der Gewichtsänderung, d. h. die DTG-Kurve erhält man durch den durch die Bewegung des Balkens in der magnetischen Spule induzierten Strom, der proportional der Bewcgungsgeschwindigkeit ist. Die die Enthalpieänderung darstellende DTA-Kurve, die mit Hilfe zweier gegeneinander geschalteter Thermoelementpaare erhalten wird, ist der zwischen der Prüfsubstanz und dem ebenfalls in den Ofenraum gesetzten Inertstoff bestehenden Temperaturdifferenz proportional. Die erwähnten drei Kurven und die Probentemperatur werden auf einer rotierenden Walze auf lichtempfindlichem Papier automatisch registriert. Der Heizstrom des Ofens wird durch einen ToroidTransformator geregelt. Der Apparat wird von den Ungarischen Optischen Werken MOM hergestellt.

Im Laufe unserer Versuche wurden oxalat-, purin- und phosphathaltige Nieren-, Harnleiter- und Blasensteine derivatographisch untersucht und gefunden, daß gewisse Verschiedenheiten in der thermischen Zersetzung dieser Steine bestehen. Art und Spitzentemperaturen derDTAund DTG-Kurven sind kennzeichnend für die Zusammensetzung der Steine, während die TG-Kurve eine quantitative Auswertung gestattet.

In Abbildung 2 sieht man die Derivatogramme von analysenreinem Calciumoxalat (links) und eines aus Calciumoxalat-Monound Dihydrat bestehenden Harnleitersteines (rechts). Aus der untersten TG-Kurve sieht man, daß die Zersetzung in drei Stufen erfolgt: Zuerst entweicht das Kristallwasser, dann verwandelt sich das Calciumoxalat unter Kohlenoxydverlust in Calciumcarbonat, endlich das Calciumcarbonat in Calciumoxyd. Aus der Höhe der Zersetzungsstufen kann mit Hilfe der chemischen Formeln die Menge des Calciumoxalats bzw. Monohydrats ermittelt werden. Die DTG-Kurve ist .eigentlich die Derivierte der vorigen Kurve; sie zeigt, wo die Zersetzung mit maximaler Géschwindigkeit verläuft. Die DTA-Kurve zeigt zwei Spitzen nach unten, die endotherme Vorgänge (Wasser und $\mathrm{CO}_{2}$-Verlust) bedeuten. Die Spitze nach oben stellt einen exothermen Vorgang, die Verbrennung von Kohlenoxyd dar:

Der Nierenstein enthält adsorptiv gebundenes Wasser, .zweierlei Kristall-Wasser und Matrix. Man sieht im Diagramm, daß zuerst das adsorbierte Wasser entweicht, dessen Menge unmittelbar durch die TG-Kurve gegeben wird. Bei steigender Temperatur entweicht zuerst das lockerer gebundene Kristallwasser des Dihydrats

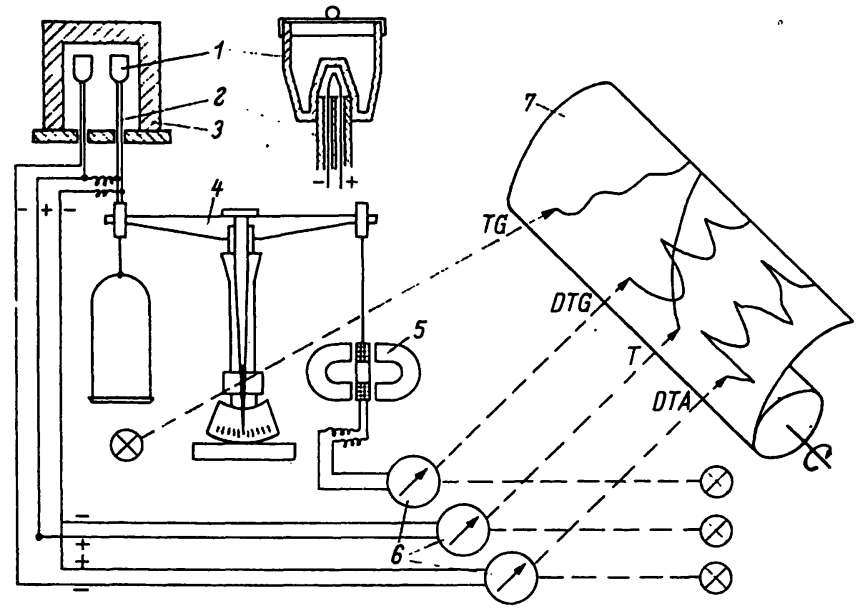

Abb. 1. Derivatograph

1: Probebehälter; 2: Thermoelement; 3: Ofen; 4: Analytische Waage; 5: Magnetspule; 6: Drehspulinstrumente mit Lichtzeiger; 7: Registrierwalze mit Photopapier; $\otimes:$ Lichtquellen

Calciumoxalat-monohydrot

Harnstein: Mischung von

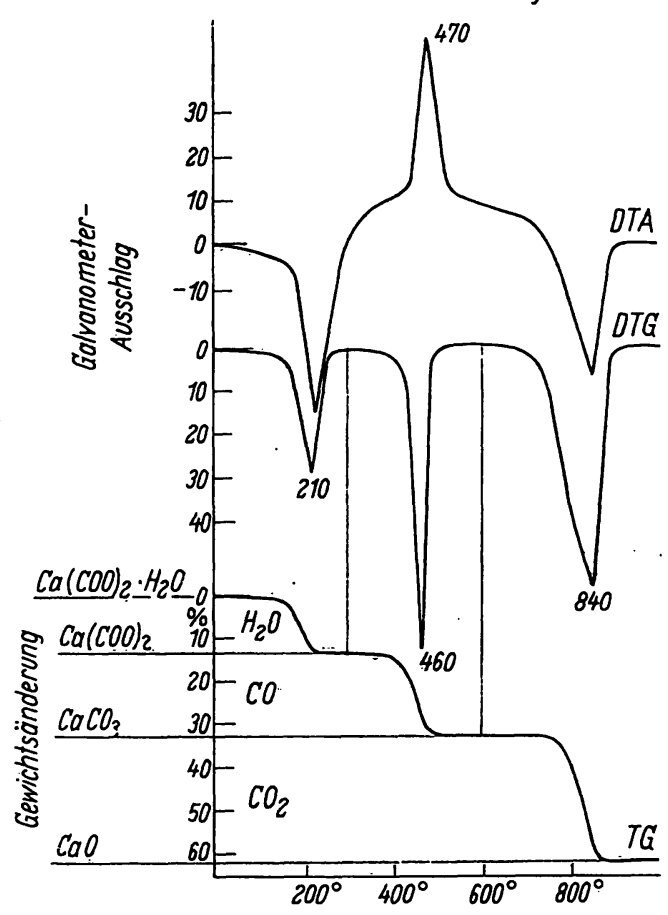
Calciumoxalat-mono- und -dihydrat

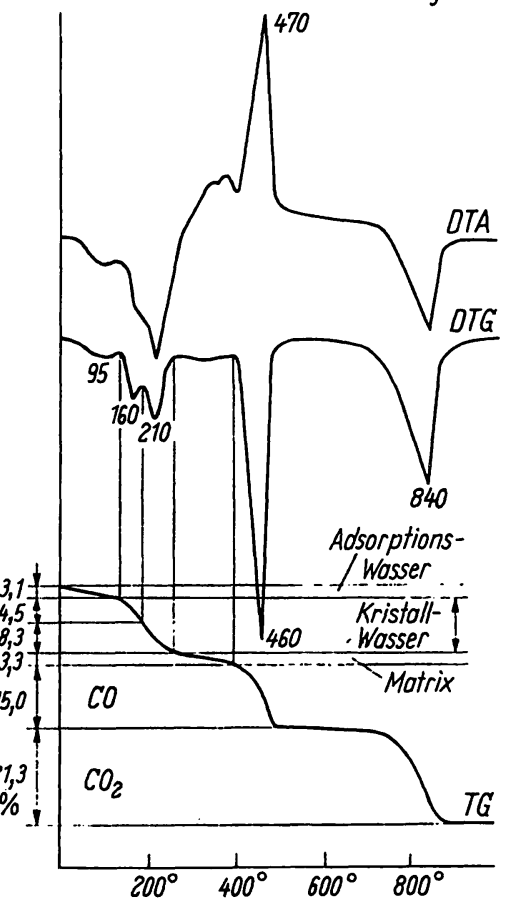

$200^{\circ} 400^{\circ} 600^{\circ} 800^{\circ}$

zwischen $140^{\circ}$ und $180^{\circ}$, dann, zwischen $180^{\circ}$ und $260^{\circ}$ das im Dihydrat zurückgebliebene Restwasser gemeinsam mit dem Kristallwasser des Monohydrats. Wie zu sehen ist, kann mit Hilfe der derivierten Kurve die quantitative Auswertung der Vorgänge leicht vorgenommen werden. Mit Hilfe der stöchiometrischen Faktoren läßt sich aus dem durch das Diagramm gegebenen Gewichtsverlust der prozentuale Mono- und Dihydratgehalt des Steines errechnen. Es ist anzumerken, $\mathrm{da} 3$ keine chemische Methode existiert, mit welcher man die auf zweierlei Weise gebundenen Wasserarten unterscheiden könnte; deshalb kann das gemeinsam auftretende Mono- und Dihydrat mit chemischen Methoden nicht genau bestimmt werden. 


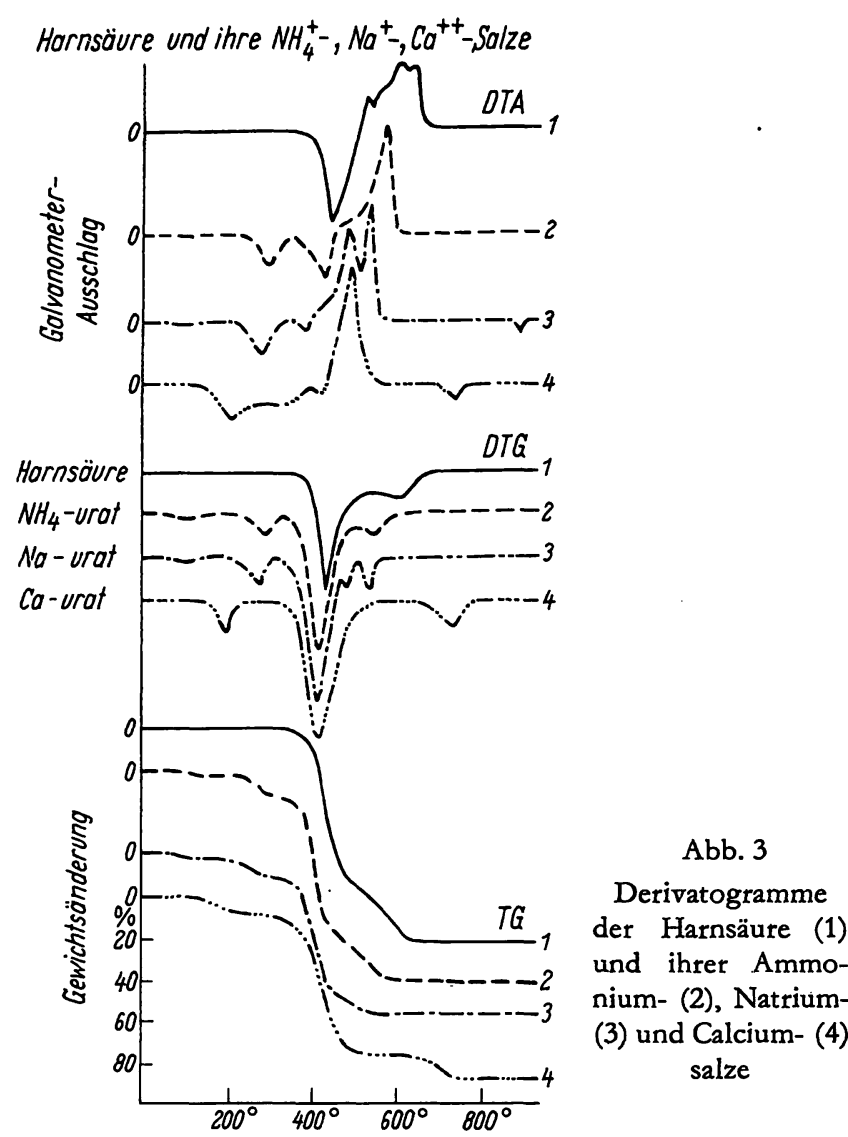

bei $260^{\circ}$. Das Verbrennungsprodukt des so entstandenen wasserfreien Natriumsalzes ist Natriumkarbonat, dessen Schmelzpunkt die DTA-Spitze bei $860^{\circ}$ zeigt. Das Calciumsalz verliert sein Kristallwasser bei $220^{\circ}$, infolge der Verbrennung entsteht Calciumcarbonat, das bei $700^{\circ}$ zu Calciumoxyd und Kohlendioxyd zerfällt.

Die Differenzen in den Spitzentemperaturen und Zersetzungsstufen gestatten die qualitative und quantitative Analyse von aus ähnlichen Komponenten bestehenden Genischen. In Abbildung 4 ist das Derivatogramm eines aus Magnesiumammoniumphosphathydrat und Harnsäure bestehenden alten, teilweise zerfallenen Steines dargestellt. Die für die Harnsäure charakteristischen Vorgänge wurden schon besprochen. Bei $100^{\circ}$ zeigen die DTG- und DTA-Kurven den Kristallwasserverlust,

Zwischen $260^{\circ}$ und $390^{\circ}$ zeigt Calciumoxalat keine Änderung; hier verbrennen die organischen Komponenten des Steines, ihre Menge liest man unmittelbar ab. Danach verläuft die Zersetzung des Calciumoxalats unter Entweichung von Kohlenmonoxyd. Die Verbrennung des letzteren gibt sich als eine exotherme Spitze bekannt. Darauf folgt eine gewichtskonstante Strecke, worauf das Calciumcarbonat zu Calciumoxyd und Kohlendioxyd zerfällt. Diese Werte gestatten eine Kontrolle der Mono- und Dihydratbestimmung. Aus dem Rückstand kann man unter Rücksicht auf die schon erhaltenen Daten auf die Menge des gegebenenfalls anwesenden Phosphats schließen.

Es wurden auch Steine von Purinstruktur untersucht. In Abbildung 3 sind nebeneinander die Derivatogramme der Harnsäure und ihrer Ammonium-, Calcium- und Natriumsalze dargestellt. Die Zersetzungskurven stimmen miteinander darin überein, daß der Purinanteil im Temperaturgebiet zwischen $320^{\circ}$ und $600^{\circ}$ in zwei Stufen verbrennt. Die maximale Geschwindigkeit des Vorgangs liegt gemäß der DTG-Kurve zwischen $400^{\circ}-430^{\circ}$, hier entweicht der größte Teil der Substanz. Darauf folgt bis $600^{\circ}$ die exotherme Verbrennung der Crackprodukte. Zur Unterscheidung geeignete Zersetzungsstufen findet man unter $300^{\circ}$ bzw. über $600^{\circ}$. Die Harnsäure enthält kein Kristallwasser; sie ist bis $300^{\circ}$ abgesehen vom Verlust etwaigen Feuchtigkeitsgehaltes gewichtsbeständig. Das Ammoniumsalz verliert hingegen Ammoniak. Die Höchstgeschwindigkeit dieses Vorgangs liegt bei $300^{\circ}$. Im weiteren verhält sich die Verbindung als Harnsäure, d. h. sie verbrennt ohne Rückstand. Das Natriumsalz verliert sein Kristallwasser

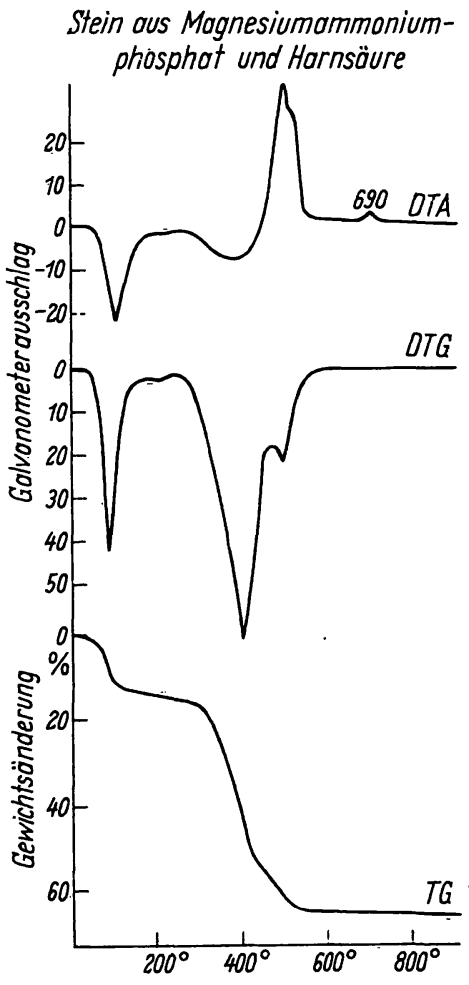

bei $690^{\circ}$ sieht man einen exothermen Vorgang ohne Geurichtsänderung, er bedeutet die Bildung von Magnesiumpyrophosphat. Wir untersuchten auch die Kusven der anderen Komponenten von in der Literatur beschriebenen Phosphatsteinen. Die Derivatogramme zeigen genügend Differenzen um eine Analyse solcher Steine auf diese Weise unternehmen zu können.

Zur thermoanalytischen Prüfung sind 50-60 mg der Substanz nötig, die Zeitdauer der Aufnahme beträgt ungefähr 100 Min. Zur Deutung der Vorgänge wurden zahlreiche Modellversuche unternommen.

Während durch chemische Analyse höchstens eine Element- bzw. Anionenzusammensetzung erhalten werden kann, lassen sich derivatographisch auch die kristallinen Komponenten von Nierensteinen ermitteln. Dies ist ein deutlicher Vorteil letzterer Methode. Die automatische derivatographische Steinanalyse liefert folglich verläßlichere Informationen, als die bisherige klinische Routineanalyse.

Dr. Ing. G. Liptay, Budapest XI/Ungarn, Egri J. U. 20=22 